\title{
Vascularization of the Enamel Organ in Developing Molar Teeth of Rats - Scanning Electron Microscope Study of Corrosion Casts -
}

\author{
By
}

\section{Shigemitsu YOSHIDA, Hayato OHSHIMA and Shigeo KOBAYASHI}

\author{
2nd Department of Oral Anatomy, Niigata University School of Dentistry, \\ 2-5274 Gakkocho-dori, Niigata 951, Japan \\ (Chief: Prof. S. Kobayashi)
}

- Received for Publication, January 28, 1989-

\begin{abstract}
Key words: Vasculature, Enamel organ, Neovascularization, Tooth development, Vascular cast
Summary: Vascularization of the enamel organ was demonstrated in the developing upper first molar teeth of rats from the 19-day embryo to 5 days after birth employing the vascular casting/ scanning electron microscope method. Capillaries were first observed in the enamel organ at the 21-day embryo. By that time, with the beginning of differentiation of the inner enamel epithelium into ameloblasts, mesenchymal cells situated in close proximity to the inner enamel epithelium had begun to differentiate into odontoblasts, but deposition of organic substances had not commenced. The occurrence of blood capillaries before the nutritional supply through the dental papilla was interrupted by the deposition of dentin and enamel, may possibly be due to the high nutritional requirements of the ameloblasts following differentiation from the inner enamel epithelium. With the advance of dentin and enamel formation, many capillaries entered the enamel organ and finally formed a flattened vascular network next to the stratum intermedium. These findings suggest that the capillaries in the enamel organ should be regarded as a change which affords a rapid and sufficient supply of metabolic substances necessary for amelogenesis. The newly developed capillaries in the enamel organ grew first by sprouting and later by loop formation.
\end{abstract}

Vascularization of the enamel organ was first described by Addison and Appleton $(1922)^{1)}$ in the developing molar teeth of rats. Subsequently, many researches have investigated the blood capillaries of the enamel organ in various mammals $2,410,15,18,19)$. However, the three-dimensional microvascular architecture of the enamel organ has not yet been fully elucidated.

The purpose of the present study was to clarify the three-dimensional microvasculature of the rat enamel organ employing the vascular casting/scanning electron microscope method introduced by Taniguchi et al. $\left(1952^{16)}, 1955^{17)}\right)$ and modified by Murakami
$(1971)^{11)}$

\section{Materials and Methods}

Young pregnant female Wistar rats were used. The onset of pregnancy was determined by vaginal smearing. The day of finding sperm in the smear was designated as day 0 in the dating of all embryos, and the day of birth was designated as day 0 in the dating of all infants. In this way, day 19 and 21 embryos and day 1 and 5 infants were obtained.

The animals were anesthetized by intraperitoneal injection of pentobarbital sodium 
and perfused through the ascending aorta with Ringer solution followed by $4 \%$ paraformaldehyde in 0.1 M phosphate buffer ( $\mathrm{pH}$ 7.4). Mercox (Dainippon-ink Co., Ltd.), which had been diluted with $25 \%$ monomeric methyl methacrylate ester ${ }^{13)}$, was then injected under moderate pressure (ca. $10 \mathrm{ml} / \mathrm{min}$ ). After the animals had been left at room temperature for $5 \mathrm{hrs}$ to allow the resin to polymerize, the upper jaws were removed with a razor blade. They were digested in $20 \% \mathrm{KOH}$ at $40^{\circ} \mathrm{C}$ for 24-48 hrs and washed thoroughly in running tap water for $12 \mathrm{hrs}$. The cast specimens were then freeze-cracked with a razor blade and freeze-dried. After drying, the casts were mounted on specimen holders with a conductive silver paint (Dotite), coated with gold in an ion sputter coater (IB-3, Eiko) and examined under a scanning electron microscope (S-510, Hitachi) at an accelerating voltage of 5-10 KV.

For light microscopy, some animals were injected with India ink, decalcified in PlankRychlo solution, sectioned at from 100 to 150 microns in thickness with a freezing microtome and mounted unstained. Paraffin and epoxy resin embedded sections were also prepared in the usual manner.

\section{Results}

In the 19-day embryo, the tooth germ was at the early bell stage of tooth development. The inner and outer enamel epithelium, stellate reticulum, stratum intermedium, dental papilla and dental follicle could be clearly distinguished, whereas no differentiation into ameloblasts or odontoblasts had yet taken place (Fig. 1a). At this stage, the enamel organ received its nutritional supply through the capillaries of the outer enamel epithelium and dental papilla (Fig. 1b). These were interconnected with each other in the cervical region of the enamel organ and formed a flattened capillary network around it. However, no blood capillaries were observed to enter the enamel organ (Fig. 1c).

In the 21-day embryo, with the beginning of differentiation of the inner enamel epithelium into ameloblasts, mesenchymal cells situated in close proximity to the inner enamel epithelium began to differentiate into odontoblasts, but deposition of organic substances had not yet commenced (Fig. 2a). Some capillaries began to enter the enamel organ in the cuspal region (Fig. 2b). These newly developed capillaries in the enamel organ grew by sprouting (Fig. 2c).

By the 1st day after birth, dentinogenesis and amelogenesis had already commenced in the cuspal region (Fig. 3a), where many capillaries were found in the enamel organ with interruption of the nutritional supply through the dental papilla by the deposition of organic substances of dentin and enamel. However, no blood capillaries were observed in the cervical region of the enamel organ, where deposition of dentin and enamel had not yet begun (Fig. 3b). The capillaries in the enamel organ formed many hairpin-like loops towards the ameloblasts or inner enamel epithelium (Fig. 3c).

By 5 days after birth, amelogenesis and dentinogenesis had progressed in all regions of the tooth crown. The capillaries in the enamel organ became increased in number and reached next to the stratum intermedium (Figs. 4a, 4b), where they formed a flattened vascular network (Fig. 4c).

\section{Discussion}

Vascularization of the rat molar enamel organ was first described by Addison and Appleton (1922) ${ }^{1)}$ based on light microscopy. They reported that capillaries were found prior to the beginning of amelogenesis. Subsequently, Glasstone $(1960)^{5)}$, Decker $(1967)^{4)}$ 
and Yoshida et al. $\left(1984^{18)}, 1985^{19)}\right)$ observed blood capillaries in the enamel organ prior to deposition of dentin and enamel, while Bernick $(1960)^{2)}$ and Tasumi (1967) ${ }^{15)}$ reported that the capillaries penetrated the enamel organ at the beginning of the appositional stage of tooth development.

Capillaries in the rat enamel organ were also confirmed in the present study. The appearance of the capillaries in the enamel organ was at the 21-day embryo and prior to the beginning of dentin and enamel formation as reported by Glasstone ${ }^{5)}$, Decker ${ }^{4)}$ and Yoshida et al. ${ }^{18,19)}$. The occurrence of capillaries before the nutritional supply through the dental papilla was interrupted by the deposition of dentin and enamel, may possibly be due to the high nutritional requirements of the ameloblasts following differentiation from the inner enamel epithelium.

With the advance of dentin and enamel formation, many capillaries entered the enamel organ and finally formed a flattened vascular network next to the stratum intermedium. These findings suggest that the capillaries in the enamel organ should be regarded as a change which affords a rapid and sufficient supply of metabolic substances necessary for amelogenesis, and also imply an important role for the stratum intermedium in enamel formation.

Two different patterns in the growth of the newly developed capillaries are known $^{3,12,14,20)}$ : one is sprouting and the other is loop formation. Schoefl and Majno $(1964)^{14)}$ speculated that these two different patterns could be interpreted as expressions of the rate of vascular growth, loops being a mode of slow expansion in response to a relatively mild stimulus, and sprouts representing a more rapid vascular growth under a stronger stimulus.

The process of entry of capillaries into the enamel organ has been described as "inva- sion" or "penetration" by many investigators employing light microscopy. However, Decker ${ }^{4)}$ and Yoshida et al. ${ }^{19)}$ clearly demonstrated by transmission electron microscopy that the capillaries in the enamel organ resulted from "indentation" or "invagination" of the outer enamel epithelium in association with their growth and were not the result of direct invasion into the stellate reticulum by penetration through the layer of the outer enamel epithelium.

In the present study, newly developed capillaries in the enamel organ were found to grow first by sprouting and later by loop formation. It is suggested that strong forces are required for the capillaries in the initial stage of indentation or invagination of the outer enamel epithelium.

\section{References}

1) Addison, W.H.F. and Appleton, J.L. Jr.: The vascularity of the enamel-organ in the developing molar of the albino rat. Am. J. Anat., 31: 161-189, 1922.

2) Bernick, S.: Vascular supply to the developing teeth of rats. Anat. Rec., 137: 141-151, 1960.

3) Cogan, D.G.: Corneal vascularization. Invest. Ophthal., 1: 253-261, 1962.

4) Decker, J.D.: The development of a vascular supply to the rat molar enamel organ. An electron microscopic study. Arch. Oral Biol., 12: 453-458, 1967.

5) Glasstone, S.: Vascularization of enamel organ in the rabbit and some rodents. Odont. Tidsk., 70: 80-87, 1960.

6) Goto, T.: On the distribution of the blood capillaries in the oral cavity and its surroundings. Part 4. Teeth and its surroundings of the dog. Hiroshima Igaku 11: 550-561, 1958. (in Japanese).

7) Jordan, H.E.: The significance of the blood vessels within the enamel organ of the molar teeth of the albino rat. Anat. Rec., 25: 291-300, 1923.

8) Jump, E.B.: Vascularity of the human enamel organ. J. Dent. Res., 17: 505-518, 1938.

9) Kingery, H.M.: The blood supply of the enamel organ in developing molar teeth of mammals. Am. J. Anat., 33: 175-195, 1924. 
10) Liao, C.T., Tada, I., Takagi, M., Higashi, Y. and Oda, M.: The vascularity of the enamel organ in the developing tooth germ of the albino mouse. J. Osaka Dent. Univ., 12: 15-31, 1978.

11) Murakami, T.: Application of scanning electron microscope to the study of the fine distribution of the blood vessel. Arch. Histol. Jap., 32: 447-454, 1971.

12) Nobuto, T., Nishikawa, Y., Hasegawa, Y., Tanda, H., Yanagihara, K., Ohnishi, K., Imai, H. and Yamaoka, A.: Experimental study on the wound healing following the periodontal surgical procedures. Japan. J. Conserv. Dent., 30: 1079-1094, 1987. (in Japanese).

13) Ohtani, O. and Murakami, T.: Peribiliary portal system in the rat liver as studied by the injection replica scanning electron microscope method. SEM/1978/II, 241-244, SEM Inc., AMF O'Hare, 1978.

14) Schoefl, G.I. and Majno, G.: Regeneration of blood vessels in wound healing. Advance in Biology of Skin, 5: 173-193, 1964.

15) Tasumi, M.: The vascular system in the deve- loping molar teeth of the rat. Tokyo Med. Dent. Univ., 14: 123-139, 1967.

16) Taniguchi, Y., Ohta, Y. and Tajiri, S.: New improved method for injection of acrylic resin. Okajimas Folia Anat. Jpn., 24: 259-267, 1952.

17) Taniguchi, Y., Ohta, Y., Tajiri, S., Okano, H. and Hanai, H.: Supplement to new improved method for injection of acrylic resin. Okajimas Folia Anat. Jpn., 27: 401-406, 1955.

18) Yoshida, S.: Changes in the vasculature during tooth eruption of the rat molar. Jpn. J. Oral Biol., 26: 94-115, 1984. (in Japanese).

19) Yoshida, S., Takeuchi, K., Hoshino, M., Yamamoto, T. and Kobayashi, S.: Ultrastructure research on the vascularization of the enamel organ in the developing molar teeth of the rat. Jpn. J. Oral Biol., 27: 549-561, 1985. (in Japanese).

20) Yoshizuka, M.: Neovascularization process in the corneal stroma after the local application of heat. Kurume Med. J., 42: 1-16, 1979. (in Japanese). 
PLATES 


\section{Explanation of Figures}

\section{Plate I}

Fig. 1. 19-day embryo.

1a: The inner and outer enamel epithelium, stellate reticulum, stratum intermedium, dental papilla and dental follicle can be clearly distinguished, whereas no differentiation of ameloblasts or odontoblasts has yet taken place. EO: enamel organ, DP: dental papilla. Azan stain. $\times 120$.

1b: The enamel organ is surrounded by capillaries of the outer enamel epithelium and dental papilla. No blood capillaries are seen entering the enamel organ. EO: enamel organ, DP: dental papilla. India-ink injection. $\times 75$.

1c: The capillaries of the outer enamel epithelium and dental papilla form a flattened network around the enamel organ. EO: enamel organ, DP: dental papilla. 
Plate I

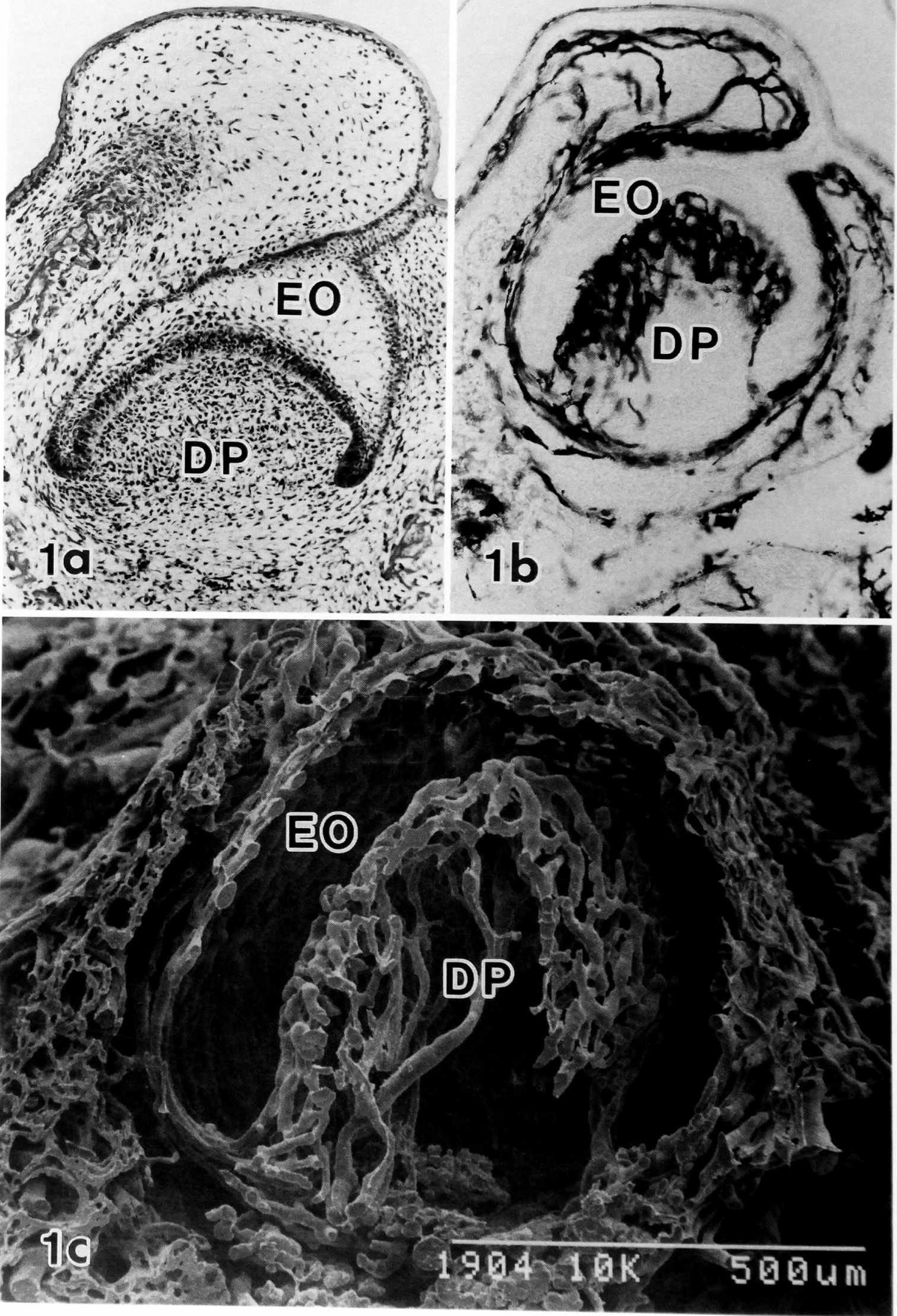




\section{Plate II}

Fig. 2. 21-day embryo.

2a: Differentiation of the inner enamel epithelium into ameloblasts and of mesenchymal cells situated in close proximity to the inner enamel epithelium into odontoblasts has taken place, but deposition of organic substances has not yet commenced. EO: enamel organ, DP: dental papilla. Azan stain. $\times 120$.

2b: Some capillaries begin to enter the enamel organ. EO: enamel organ, DP: dental papilla. India-ink injection. $\times 75$.

2c: The newly developed capillaries in the enamel organ grow by sprouting. EO: enamel organ, DP: dental papilla. 
Plate II

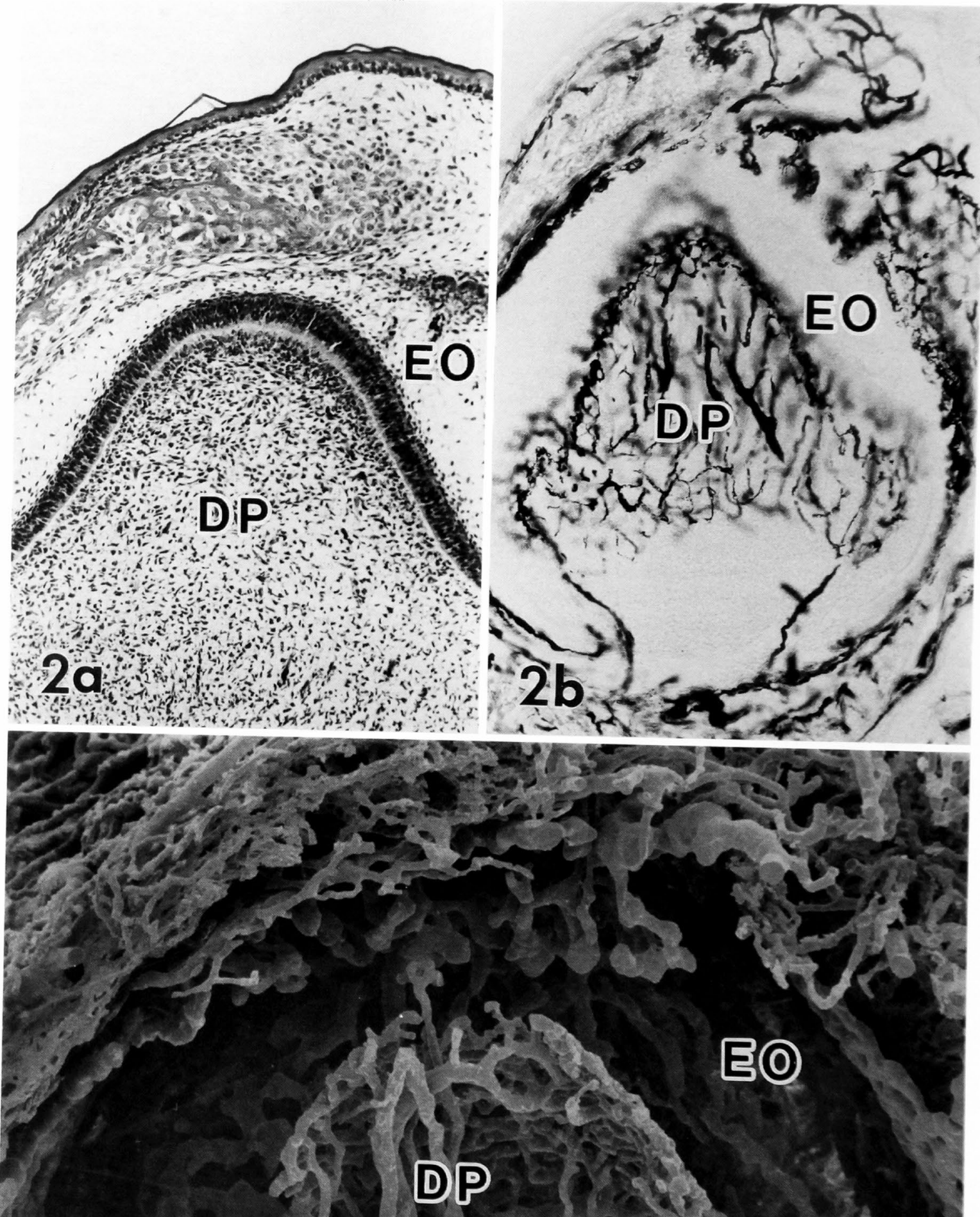




\section{Plate III}

Fig. 3. 1st day after birth.

3a: Dentinogenesis and amelogenesis have already commenced in the cuspal region. Capillaries (C) in the enamel organ approach the stratum intermedium (SI). OEE: outer enamel epithelium, SR: stellate reticulum, AB: ameloblast, E: enamel, D: dentin. Toluidine blue stain. $\times 750$.

3b: Many capillaries are found in the cuspal region, but no blood capillaries are observed in the cervical region of the enamel organ. EO: enamel organ, DP: dental papilla. India-ink injection. $\times 75$.

3c: The capillaries in the enamel organ form many hairpin-like loops (Arrow). EO: enamel organ. 
Plate III

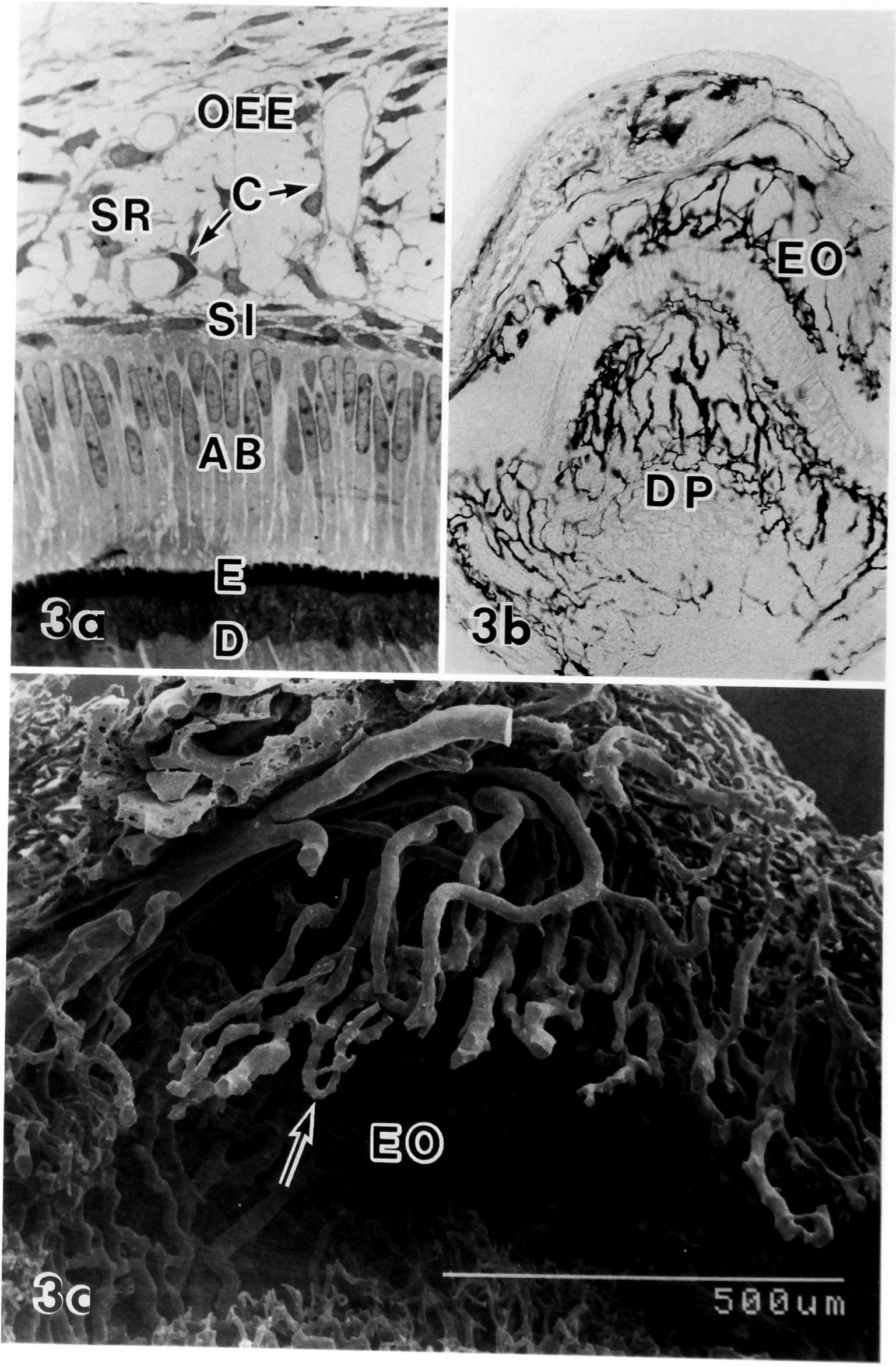




\section{Plate IV}

Fig. 4. 5 days after birth.

4a: Capillaries $(C)$ in the enamel organ are situated next to the stratum intermedium (SI). SR: stellate reticulum, AB: ameloblast, E: enamel, D: dentin. Toluidine blue stain. $\times 600$.

4b: Many capillaries are observed in the enamel organ. EO: enamel organ, DP: dental papilla. Indiaink injection. $\times 70$.

4c: The capillaries in the enamel organ form a flattened vascular network. EO: enamel organ. 
Plate IV

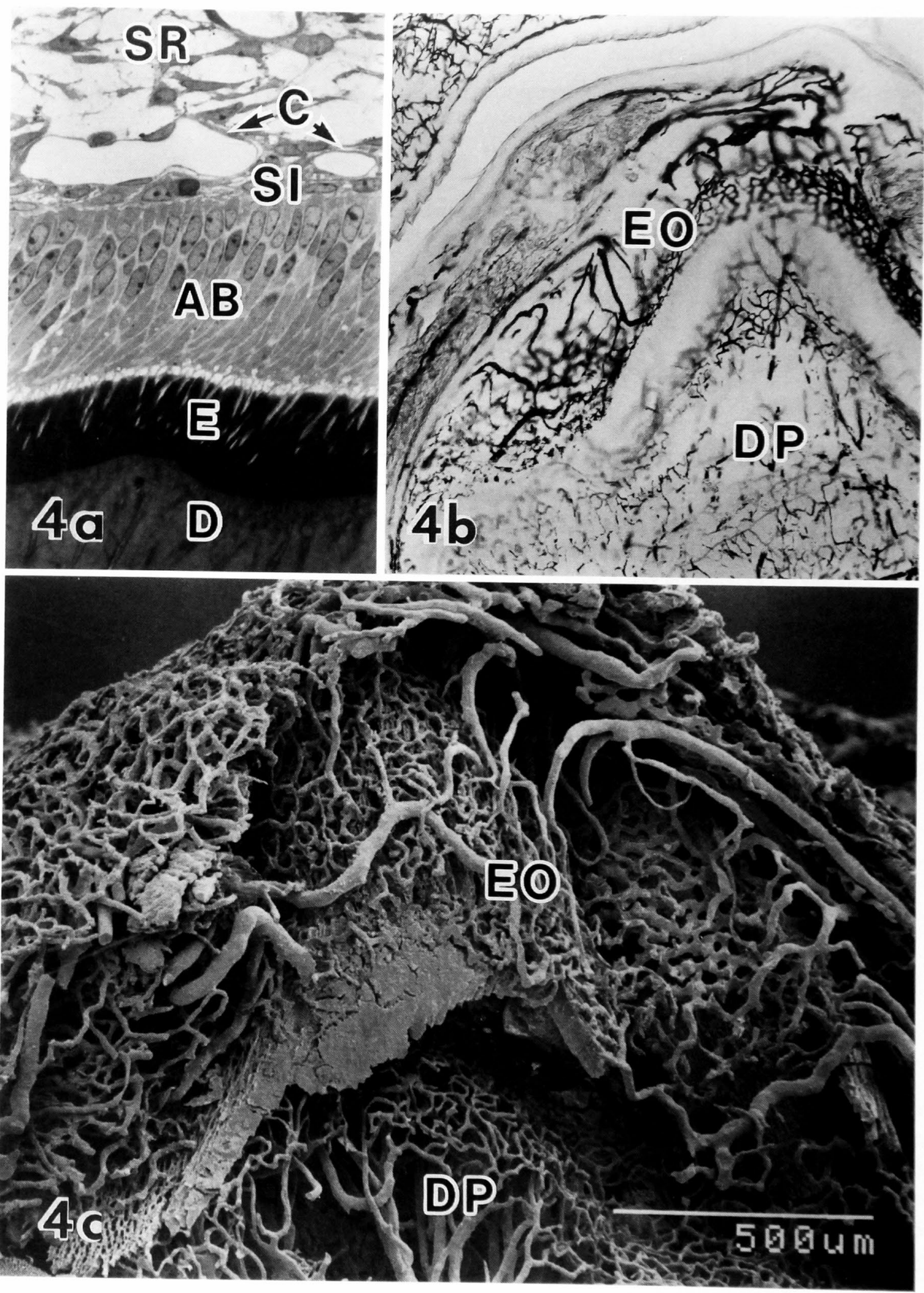

